\title{
Exploiting Semantic Term Relations in Text Summarization
}

\author{
Kamal Sarkar, Jadavpur University, India \\ (iD) https://orcid.org/0000-0002-0689-3976 \\ Santanu Dam, Netaji Subhas Open University, India
}

\begin{abstract}
The traditional frequency-based approach to creating multi-document extractive summary ranks sentences based on scores computed by summing up TF*IDF weights of words contained in the sentences. In this approach, TF or term frequency is calculated based on how frequently a term (word) occurs in the input, and TF calculated in this way does not take into account the semantic relations among terms. In this paper, the authors propose methods that exploit semantic term relations for improving sentence ranking and redundancy removal steps of a summarization system. The proposed summarization system has been tested on DUC 2003 and DUC 2004 benchmark multi-document summarization datasets. The experimental results reveal that performance of the multi-document text summarizer is significantly improved when the distributional term similarity measure is used for finding semantic term relations. The multi-document text summarizer also outperforms some well-known summarization baselines to which it is compared.
\end{abstract}

\section{KEYWORDS}

Distributional Semantics, Hybrid Similarity, Multi-Document Text Summarization, Redundancy, Semantic Similarity, Word Embedding, Wordnet-Based Semantics

\section{INTRODUCTION}

Information overload is a critical problem on the Internet. For managing information load on the Internet, one of the most effective mechanisms is text summarization. Text summarization reduces the input document(s) to a summary which is a condensed version of the input. Summarization can help users in many ways-(1) it enables readers to quickly understand what the document(s) is about, (2) summaries can be presented with the search results to help users in understanding whether the linked documents are relevant or not, (3) bandwidth can be saved if instead of sending the whole document, a summary is sent first to the small screen devices. Summaries are also useful in many other applications such as text clustering and classification. Though many researchers have been trying to find solutions to text summarization problem for the last many years, there is further scope for doing research for finding better solution to the problem. The reason is that text summarization is a human ability which is very difficult to model. 
According to the previous research works (Goldstein et al., 2000; Gupta \& Siddiqui, 2012; Sarkar, 2014; Sarkar, 2009a), summary can be of two types: extract and abstract. An extract is a summary created by selecting text segments (sentences) from the input whereas an abstract is a summary which is created by reformulation of text segments selected from the summary. An abstract may contain some words which are not present in the input. The most existing abstractive summarization methods deal with generating very short or ultra-summary (Sarkar \& Bandyopadhyay, 2005; Zajic, Dorr \& Schwart, 2002; Rush, Chopra \& Weston, 2015; Nallapati et al.,2016; Nallapati, Zhai, \& Zhou, 2017). In this paper, we focus on generating extractive multi-document summaries. Multi-document summaries are relatively longer than very short summaries.

The most previous works on extraction based summarization are sentence ranking based. The sentence ranking based approach ranks sentences based on scores where score of a sentence is calculated by combining various feature based scores such as frequency of terms, sentence position and/or cue phrases (Luhn, 1959; Sarkar, 2009b; Sarkar, Nasipuri \& Ghosh, 2011). After ranking sentences, the top $n$ sentences are chosen based on the compression ratio.

Centroid based summarization approach (Radev et al., 2004) is also an extractive approach to multi-document summarization that ranks sentences based on their similarities to the centroid which is created by choosing a set of most important words from the input cluster of documents. Here word importance is measured by the TF*IDF weight. In this approach, calculation of TF or term frequency is based on how frequently a term (word) occurs in the input.

Not only the centroid-based approach, but many other summarization approaches also use term frequency calculation which considers syntactic term matching without taking into account the semantic relations among terms. Due to this problem, the traditional TF*IDF based sentence-ranking approach places some summary worthy sentences in the rank positions far from the top ranked sentences and so, those sentences are not selected into the summary due to a predefined summary length restriction.

Since the input to a multi-document summarizer is a set of related documents, a multi-document summary may contain redundancy. Redundancy is one of the crucial factors in multi-document summarization because redundant information in a summary makes the summary less informative. Maximal marginal relevance is a popular technique for redundancy removal while selecting top $n$ sentences for creating an extract (Carbonell \& Goldstein, 1998). Maximal marginal relevance technique uses cosine similarity between sentences for identifying similar sentences where each sentence is represented using TF*IDF based Bag-of-words model. Hence term mismatch problem leads to data sparseness problem which affects redundancy removal performance.

So, other than above mentioned cases, there are a number of existing extractive summarization methods (mentioned in the subsequent section) that use TF*IDF based Bag-of-Words model for text representation where a term weight is calculated using the traditional TF*IDF method.

In this paper, we present a multi-document summarization method that exploits semantic term relations in computing term weights as well as similarity between sentences. The main contributions of our work are:

- Hybridizing traditional string-matching based term weighting method with the semantic term relation-based term weighting method to calculate more accurate term weights which are used in sentence ranking step of the text summarization process.

- Incorporating semantic term relations in sentence similarity measure which is used in redundancy removal step of the text summarization process.

- Comparing WordNet based semantic similarity measure and distributional semantic similarity measure for finding semantic term relations which has been exploited in term weighting and sentence similarity computing.

- DUC 2003 and DUC 2004 benchmark multi-document summarization datasets have been used to test our proposed models. 
- We have also performed statistical significance tests to prove whether the difference of performance scores achieved by our proposed system and the well-known existing summarization baseline systems are statistically significant or not.

Organization of the paper is as follows: In next section, we present a survey of the previous works related to our work. In the subsequent sections, we present our proposed methods and the experimental results. Finally we conclude our work.

\section{RELATED WORK}

The most existing sentence extraction based multi-document summarization has two primary steps (1) sentence ranking and (2) redundancy removal. The most important feature for sentence ranking is the weights of the terms contained in a sentence (Radev et al., 2004). Though some domain specific features such as sentence position (for news domain) and cue phrases (for medical domain) (Sarkar, 2009b) are also used for improving multi-document summarization performance, the most important feature is the term weight which is traditionally computed using TF*IDF method. Here $\mathrm{TF}$ or term frequency is the average frequency of the term (total occurrences of the term in the input document-set divided by number of documents in the input document-set) and IDF (inverse document frequency) is calculated from a large corpus using the formula: $\log (\mathrm{N} / \mathrm{df})$, where $\mathrm{N}=$ total number of documents in the corpus (a large collection of documents which may not include test datasets) and df (document frequency) of a term means how many documents of the corpus contain the term at least once(Radev et al., 2004).

Other than sentence ranking, term weight is also used in redundancy removal. While summary is created incrementally using greedy approach, the redundant sentences are removed by finding the similarity between the sentence to be selected and any sentence previously selected in the summary (Radev et al., 2004).

This is known as maximal marginal relevance(MMR) approach to redundancy removal (Carbonell $\&$ Goldstein, 1998). For computing cosine similarity between sentences, the TF*IDF based bagsof-words representation is considered. This suffers from so-called data sparseness problem which hampers redundancy removal process.

Unlike the MMR based approach that addresses redundancy issue locally, clustering based approach addresses redundancy issue globally by creating the groups of similar sentences and selecting sentence one by one from the clusters to form the final summary (Hardy, 2002; Sarkar, 2009a; Hatzivassiloglou et al., 2001). Since performance of the most existing clustering methods used for sentence clustering depends on similarity measure used in finding similarity between sentences, the choice of similarity measure can influence clustering-based summarization performance.

In the graph based multi-document text summarization approach (Uçkan \& Karc1, 2020; AlSabahi \& Zuping, 2019; Belwal, Rai, Gupta, 2020; Erkan \& Radev, 2004; Mihalcea \& Tarau, 2004; Mihalcea \& Tarau, 2005; Erkan \& Radev, 2005), a node of the graph corresponds to a sentence and a pair of nodes is connected by an edge if cosine similarity between the corresponding sentences is greater than a threshold value. The most existing graph-based methods mentioned above also mainly uses the cosine similarity measure for building the similarity graph and redundancy removal.

To deal with the data sparseness problem caused by the traditional TF*IDF based bag-of-words text representation, some researchers have designed more enhanced sentence similarity measure (Sarkar, Saraf \& Ghosh, 2015) that combines cosine similarity measure with the similarity measure that uses dynamic programming based optimal local alignment between two sentences. Dam and Sarkar (2019) have applied a hybrid sentence similarity measure for improving multi-document text summarization performance. Aliguliyev (2009) has designed a sentence dissimilarity measure which is defined as a product of global and local dissimilarity measures and has shown that (dis) similarity measure has an impact on that summarization results. Most recently, Mohd, Jan and Shah (2020) 
has proposed an approach that incorporated distributional sentence similarity measure in sentence clustering-based summarization method. Song, Han and Rim (2004) have presented a new term weighting method that considers the WordNet based semantic relations (e.g. synonym, antonym, etc.) between words for term weighting applied to text summarization.

\section{Our Method}

The first step of our proposed method is the pre-processing step which includes removal of short sentences (containing less than 9 words) and removal of stop-words from the sentences of the input document set. We used a stop-word list that includes the very common terms such as articles, prepositions and common verbs. The other steps of our proposed method are: (1) Term weighting using semantic relations of a term with other terms and term frequency, (2) Sentence ranking, (3) redundancy removal using a sentence similarity measure that uses semantic term relations and (4) Summary generation. Figure 1 shows the block diagram of our proposed method.

\section{Exploiting Semantic Term Relations for Term Weighting}

Semantic relations among terms can be measured in two ways - (1) WordNet based semantic relations and (2) distributional similarity between the terms. The most common WordNet based semantic relations are synonymy, antonym, homonymy etc. On the other hand, distributional relations between two words depends on the accompany they keep, that is, contextual similarity. Since we use two different methods for finding semantic term relations, we categorize term weighting methods into two ways- (1) term weighting using WordNet based semantic relations and (2) term weighting using distributional similarity between terms.

\section{Figure 1. Block diagram of our proposed method}

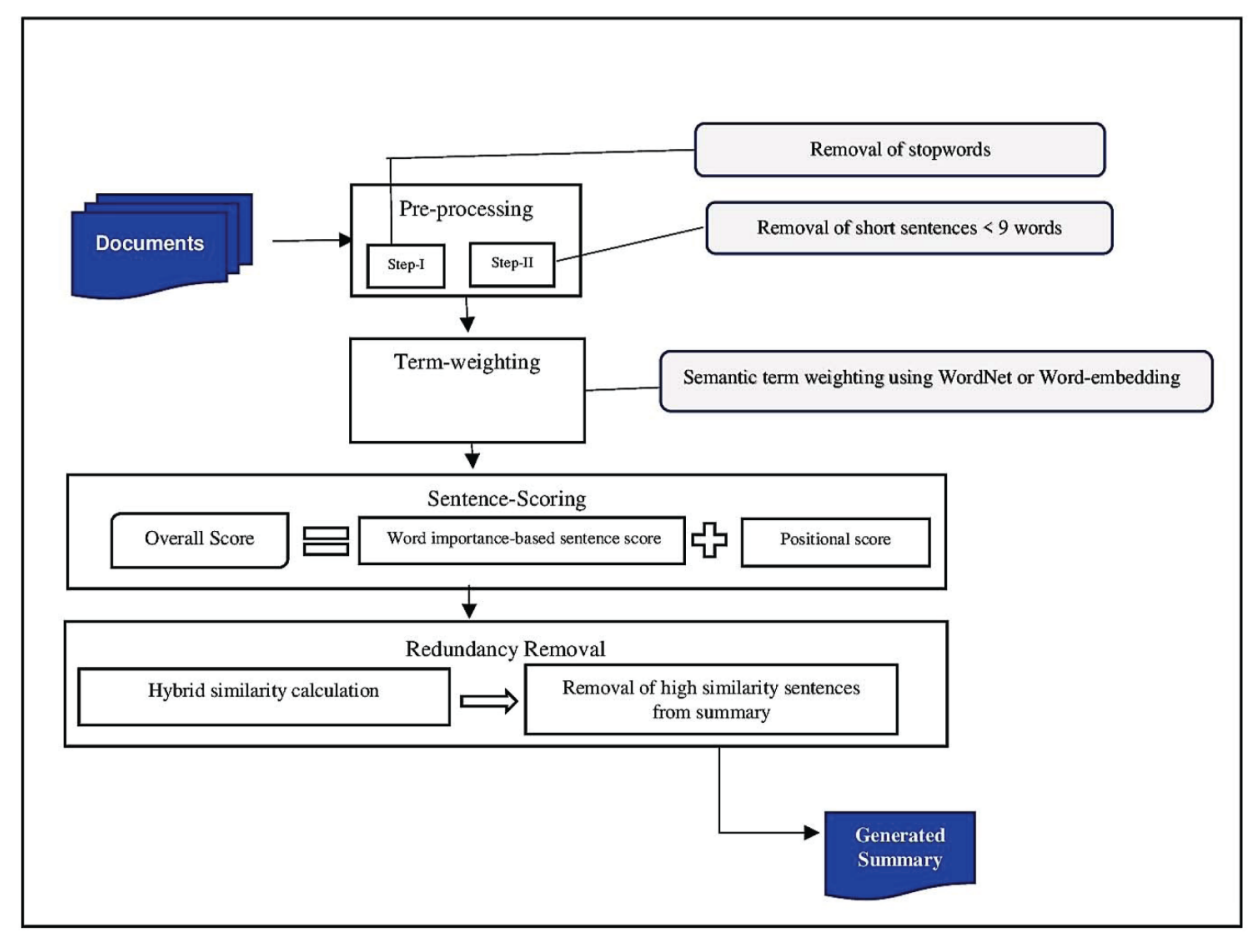




\section{Using WordNet Based Semantic Term Relations for Term Weighting}

In this term weighting scheme, we exploited lexical semantic term relations derived from a lexical network of English words called WordNet (Fellbaum,1998). The words of various lexical types such as nouns, verbs, adjectives, and adverbs are organized into a network of words which are interlinked with various relations (Budanitsky \& Hirst. 2006). WordNet maintains variety of relations between words and WordNet based term relations is a more general concept than the concept of similarity (Budanitsky \& Hirst. 2006). For example, the relation between "bank" and "trust company" is that they are synonyms and the relations between the words "hot" and "cold" is that they are antonyms. So, WordNet based term relations should be used more carefully while computing similarity between sentences. We have carefully used WordNet based semantic term relatedness measures for finding more synonymous terms. Out of existing measures such as Lin (Lin, 1998), path length (Resnik,1995), Wup (Wu and Palmer,1994), Dam and Sarkar (2019) have shown that Wup is more effective semantic relation measure. Wup similarity (Wu and Palmer,1994) between two concept elements tp and tq is defined in the following way:

$$
w u p\left(t_{p}, t_{q}\right)=\frac{2^{*} d}{L e n_{p}+L e n_{q}+2^{*} d}
$$

where $\mathrm{d}$ is the depth from the root of WordNet hierarchy to the LCS (least common subsumer), Len $n_{p}$ is the length of path from $t_{p}$ to LCS, and Len $n_{q}$ is the length of path from $t_{q}$ to LCS. LCS (least common subsumer) is the least common node on the paths corresponding to $t_{p}$ and $t_{q}$. For example, "organism" is the LCS of the two concept elements "canine" and "chap". In other words, "organism" is the lowest node common between the paths to the concept elements "canine" and "chap" from the root of the WordNet hierarchy. Here similarity between two concepts is defined by how close they are in the hierarchy. Figure 2 shows relations between two concept elements "canine" and "chap.

We have taken two concepts $\mathrm{X}$ and $\mathrm{Y}$ as semantically similar if wup(X, Y) is greater than a predefined threshold ( 0.85 in our setting). Here the threshold value has been set to relatively higher value because we have observed that choosing higher threshold on Wup value gives conceptually similar words. For example, with threshold value of 0.85 , we find that the concepts - "police", "Committee", "Commission", "Agency" are most similar with the given concept "agencies. Given two words $\mathrm{w}_{1}$ and $\mathrm{w}_{2}$, word similarity is computed by taking the maximum of Wup values computed

Figure 2. Relations between two concept elements "canine" and "chap" in the hierarchy

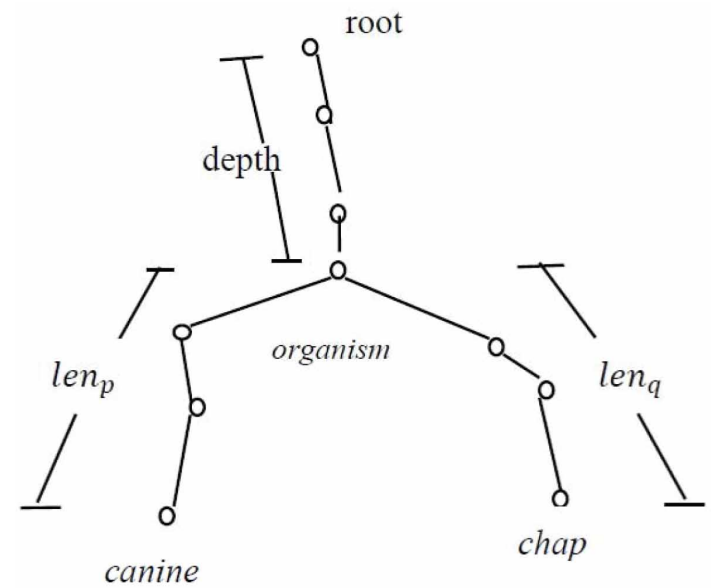


for all possible pairs of senses of $\mathrm{w}_{1}$ and $\mathrm{w}_{2}$. For finding Wup values, we have used WordNet available as a part of NLTK toolkit under Python platform.

Using the above-mentioned word similarity measure, we have computed semantic term frequency (STF) of a word. $\operatorname{STF}(w)$ is defined as how many words of the document set are semantically similar with the word $w$. While calculating $\operatorname{STF}(w)$, it does not include the count of matches where two words are string identical. Finally, weight of the word is computed as follows:

$$
\text { Weight }(w)=(S T F(w)+T F(w)) * \operatorname{IDF}(w)
$$

where:

$\operatorname{STF}(w)=$ semantic term frequency of $w$ $\operatorname{TF}(w)=$ the traditional term frequency of $w$, that is, how many times the word $w$ occurs in the document-set $\operatorname{IDF}(w)=$ Inverse Document Frequency of the word $w$

\section{Using Word Embedding Based Term Relations for Term Weighting}

Word embedding(Mikolov, 2013) refers to distributed representation of words in k-dimensional vector space where each word corresponds to point represented by k-dimensional vectors. Words which have the similar contextual usages in representing the similar concepts are transformed into the vectors closely positioned in the vector space and the words that have the same meaning are represented in the similar way by the word embedding model. In contrast to the sparse and very high dimensional one-hot vector representation for a word, the word vector produced by the word embedding model is highly dense. This dense representation improves generalization power and provides contextual similarity clues based on which semantic relatedness between the words can be measured.

Cosine similarity between the distributed vector representations of the words called word vectors is used to measure the degree of semantic relatedness between two words. Gensim word2vec model under Python platform, which is one of the popular ready-to-use word embedding models, has been used for our implementation. We have used text 8 corpus $^{1}$ for training word 2 vec model. Two important parameters of word2vec model, which need to tuned to obtain the effective word vectors are dimension of the word vector and mincount. All words with total frequency lower than mincount are ignored during model development. For our implementation, dimension of the word vector and mincount are set to 200 and 5 respectively. There are multiple training algorithms for developing word2vec model. The most common training algorithms are CBOW (Continuous Bag of words) and Skip-bigram. We have chosen Skip-bigram algorithm. The model is trained with Text8Corpus which contains about 17 million words derived from Wikipedia. After cleaning Wikipedia texts by removing URLs, punctuation, non-ASCII characters, Text8Corpus is developed for training Word2Vec model. Gensim library provides built-in functions for handling Text8Corpus. Given two-word vectors for the two different words, the semantic relatedness between the words is calculated using cosine similarity measure. If the k-dimensional word vectors for the two different words are: $\mathrm{u}_{1}=\left\{\mathrm{u}_{11}, \mathrm{u}_{12}, \mathrm{u}_{13}, \ldots, \mathrm{u}_{1 \mathrm{k}}\right\}$ and $\mathrm{v}_{2}=\left\{\mathrm{v}_{21}, \mathrm{v}_{22}, \mathrm{v}_{23}, \ldots . ., \mathrm{v}_{2 \mathrm{k}}\right\}$, the semantic relatedness between the words is calculated as follows:

$$
\text { Semantic_Sim }\left(u_{1}, v_{2}\right)=\frac{u_{1} \cdot v_{2}}{\left|u_{1}\right|\left|v_{2}\right|}
$$

Using the word embedding based semantic relatedness measure defined above, the term weight is calculated using the equation 1 with only the difference in how STF is calculated. In the present case, $\operatorname{STF}(w)$ (semantic term frequency of the word w) is calculated using the word embedding based 
semantic relatedness measure as defined in equation (2). Like the threshold as applied on WordNet based semantic relatedness value, we also choose a threshold on word embedding based semantic similarity value. If semantic similarity between two words is greater than a predefined threshold value, they are considered to have similar meanings. We have determined this threshold value through experimentations (the details are discussed in result section).

\section{Sentence Ranking}

For ranking sentences of the input document, each sentence is assigned a score which is calculated using sum of two sentence scores -(1) sentence score computed based on word importance and (2) positional score. To compute sentence score based on word importance, weights of the words contained in the sentence are summed up. While computing sentence score using word importance, words which have low weight values are not taken into consideration. Weight of a word is calculated using the formula given in the equation (1). The sentence score which is computed based on word importance is formulated in equation 3 :

$$
\operatorname{Score}_{t}(S)=\sum_{w \in S} \text { weight }(w)
$$

Other than the term weight based sentence score, the sentence positional score is also useful in text summarization of news documents. Since DUC 2003 and DUC 2004 datasets (discussed in the result section) consist of news documents, we also combine the term weight based sentence score with the positional score. The positional score for a sentence $\mathrm{S}$ is calculated as follows:

$$
\operatorname{Score}_{p}(S)=\frac{1}{\sqrt{i}}
$$

where $\mathrm{i}$ is the sentence position.

The final score for a sentence is calculated by combining term weight-based score and positional score. Before this blending, the term weight-based score is normalized by dividing the score by the maximum of scores assigned to the input sentences. The overall combined score for a sentence $S$ is calculated as follows:

$$
\text { OverallScore }(S)=\text { normalized }_{\text {Score }}(S)+\text { Score }_{p}(S)
$$

After assigning scores to the sentences, the sentences are ranked based on their combined scores computed using equation (5).

\section{Incorporating Semantic Term Relations in Redundancy Removal Process}

Redundancy removal is a crucial step in multi-document summarization task because the input to a multi-document summarizer is a cluster of documents describing the similar topics. While creating a summary, the sentences containing redundant information are discarded to allow selection of relatively more diverse sentences in the summary. A sentence is considered to be a redundant sentence if its similarity with any of the previously selected summary sentences is greater than a threshold value. So, at this stage sentence similarity measure plays an important role. A hybrid similarity measure is proposed for dealing with redundancy issue. Our proposed sentence similarity measure blends the traditional cosine similarity measure with the semantic similarity measure that exploits semantic term relations. We combined these two similarity measures because the traditional cosine similarity measure 
depends on simple term matching between two sentences and hence it misses to identify semantically related sentences. On the other hand, semantic similarity measure cannot capture relative importance of the terms in the input. So, combination of these two similarity measures gives us more accurate hybrid similarity measure which is more useful in reducing redundancy from the generated summary.

Using word level semantic similarity which can be computed using either Wup measure or word vector based distributional similarity measure (as discussed in the earlier sections), we calculate semantic similarity between sentences as follows:

$\operatorname{SemanticSentenceSim}\left(S_{1}, S_{2}\right)=\frac{2 * \# \text { Word__Match }}{\left|S_{1}\right|+\left|S_{2}\right|}$

where \#word_match is the number of words common between S1 and S2. We consider that two words are semantically matched if Wup value or their distributional similarity value is greater than a threshold value (which is determined through experimentation). Besides semantic matching, \#word_match also includes the counts of exact word matches (string identical) between two sentences. Stop words are not considered in this sentence similarity calculation.

To compute cosine similarity between two sentences, two sentences are represented as vectors of real values: $\mathrm{S}_{1}=\left\{\mathrm{w}_{11}, \mathrm{w}_{12}, \mathrm{w}_{13}, \ldots, \mathrm{w}_{1 \mathrm{n}}\right\}$ and $\mathrm{S}_{2}=\left\{\mathrm{w}_{21}, \mathrm{w}_{22}, \mathrm{w}_{23}, \ldots ., \mathrm{w}_{2 \mathrm{n}}\right\}$, where $\mathrm{w}_{\mathrm{i}, \mathrm{j}}$ is the TFS-IDF value for the jth word in the ith sentence and $n$ is the vector length which is basically the number of distinct words present in the pair of sentences (stop words are removed). The following formula (Erkan \& Radev, 2004) defines cosine similarity between $S_{1}$ and $S_{2}$ :

Cosine_Sim $\left(S_{1}, S_{2}\right)=\frac{S_{1} \cdot S_{2}}{\left|S_{1}\right|\left|S_{2}\right|}$

TFS-IDF value of a term is computed by the product of TFS and IDF, where TFS is the number of times the term occurs in the sentence and IDF is computed as follows:

$I D F_{i}=\log \left(\frac{N}{n_{i}}\right)$

where $\mathrm{N}=$ the total number of the documents in a corpus and $\mathrm{n}_{\mathrm{i}}$ is the number of documents containing word $i$ at least once. IDF value is computed on a large corpus which does not include our test datasets.

Finally, the hybrid similarity measure is a weighted combination of the above-mentioned two similarity measures:

$$
\operatorname{Hybrid}-\operatorname{Sim}\left(S_{1}, S_{2}\right)=\alpha^{*} A+(1-\alpha) * B
$$

where:

$$
\begin{aligned}
& \mathrm{A}=\text { SemanticSentenceSim }\left(S_{1}, S_{2}\right) \\
& \mathrm{B}=\text { Cosine_Sim }\left(S_{1}, S_{2}\right)
\end{aligned}
$$


For best summarization results, we tune the values of $\alpha$ and find that setting $\alpha$ to 0.6 gives the best results on the summarization datasets. Here achieving better results using higher value of $\alpha$ reveals that incorporating semantic term relations in sentence similarity measure is more effective for reducing redundancy in the summary.

\section{Summary Generation}

After ranking the sentences based on their scores, the summary is created by selecting the top ranked sentences from the ranked list. While creating a summary, the sentences containing redundant information are also discarded. A sentence is considered to be redundant sentence if its similarity with any of the previously selected summary sentences is greater than a threshold value. Here the sentence similarity is computed using the hybrid similarity measure given in equation 9 .

\section{Illustration of our Proposed Approach With an Example}

In this subsection, we will illustrate our method of summarization with an example. For simplicity reasons, we consider two documents, each contains only one sentence. The documents are shown in Table 1.

\section{Pre-Processing}

As per our proposed method, our first step is the pre-processing which includes discarding short sentences (sentences with less than equal to 9 words are discarded) and stopword removal. Since our example documents do not have any sentence $<=9$ words, no sentence is discarded and the output obtained after stopword removal is shown in Table 2.

\section{Process of Term Weighting Based on Semantic Relations of a Term With Other Terms}

In Figure 3, we have shown scores for semantic term relation between pairs of words. We have considered WordNet based term similarity score for this case study and the similarity scores whose are greater than threshold is highlighted in the figure. In the figure, the pair of words whose semantic similarity values crosses the threshold are: (Commerce, trade), (wound, hurt) and (money, currency).

\section{Sentence Scoring}

Sentence scoring is done by combining score of a sentence based on word importance (equation 1) and positional score (equation 4). Table 3 and Table 4 show TF(term frequency), STF(Semantic TF),

Table 1. Input cluster of documents

\begin{tabular}{|c|l|}
\hline Document No. & \multicolumn{1}{c|}{ Sentences } \\
\hline 1 & $\begin{array}{l}\text { The Russian crisis has hurt bilateral trade, caused the Ukrainian currency to fall as per } \\
\text { committee. }\end{array}$ \\
\hline 2 & $\begin{array}{l}\text { The Russian money market and investors assets has continued to fall since the wound hit in } \\
\text { commerce. }\end{array}$ \\
\hline
\end{tabular}

Table 2. Output obtained after stopword removal

\begin{tabular}{|c|l|}
\hline Sentence No. & \multicolumn{1}{|c|}{ Sentences } \\
\hline 1 & Russian crisis hurt bilateral trade, caused Ukrainian currency fall committee. \\
\hline 2 & Russian money market investors assets continued fall wound hit commerce. \\
\hline
\end{tabular}


Figure 3. Semantic term relation score between pair of words

\begin{tabular}{|c|c|c|c|c|c|c|c|c|c|c|c|c|c|c|c|c|c|c|}
\hline & Ukranian & caused & Commerce & Wound & Hit & Fall & Continued $\mathrm{B}$ & Bilateral & Currency & Committel & Russian & Crisis & Market & Investor & Trade & Money & Hurt & Assets \\
\hline Ukranian & 1 & 0.24 & 0.25 & 0.21 & 0.22 & $\begin{array}{l}0.25 \\
\end{array}$ & 0 & 0 & 0.25 & $\begin{array}{l}0.24 \\
\end{array}$ & 0.13 & 0.24 & 0.25 & 0.13 & $\begin{array}{l}0.24 \\
\end{array}$ & 0.25 & $\begin{array}{l}0.22 \\
\end{array}$ & 0.29 \\
\hline caused & 0.24 & 1 & 0.53 & 0.22 & 0.47 & 0.27 & 0 & 0 & 0.27 & 0.25 & 0.14 & 0.25 & 0.53 & 0.14 & 0.5 & 0.27 & 0.24 & 0.31 \\
\hline \begin{tabular}{|l|} 
Commerce \\
\end{tabular} & 0.25 & 0.53 & 1 & 0.24 & 0.59 & 0.29 & 0 & 0 & 0.29 & 0.27 & 0.15 & 0.27 & 0.67 & 0.15 & 0.94 & 0.29 & 0.25 & 0.33 \\
\hline Wound & 0.21 & 0.22 & 0.24 & 1 & 0.21 & 0.24 & 0 & 0 & 0.24 & 0.22 & 0.12 & 0.56 & 0.24 & 0.12 & 0.22 & 0.24 & 0.95 & 0.4 \\
\hline Hit & 0.22 & 0.47 & 0.59 & 0.21 & 1 & 0.25 & 0 & 0 & 0.25 & 0.24 & 0.13 & 0.24 & 0.62 & 0.13 & 0.56 & 0.25 & 0.22 & 0.29 \\
\hline Fall & 0.25 & 0.27 & 0.29 & 0.24 & 0.25 & 1 & 0 & 0 & 0.43 & 0.27 & 0.15 & 0.27 & 0.29 & 0.15 & 0.27 & 0.43 & 0.25 & 0.33 \\
\hline Continued & 0.15 & 0.17 & 0.18 & 0.14 & 0.15 & 0.18 & 1 & 0.4 & 0.18 & 0.17 & 0.18 & 0.17 & 0.18 & 0.18 & 0.17 & 0.18 & 0.15 & 0 \\
\hline Bilateral & 0 & 0 & 0 & 0 & 0 & 0 & 0 & 1 & 0 & 0 & 0 & 0 & 0 & 0 & 0 & 0 & 0 & 0 \\
\hline Currency & 0.25 & 0.27 & 0.29 & 0.24 & 0.25 & 0.43 & 0 & 0 & 1 & 0.27 & 0.15 & 0.27 & 0.29 & 0.15 & 0.27 & 0.86 & 0.25 & 0.33 \\
\hline Committee & 0.24 & 0.25 & 0.27 & 0.22 & 0.24 & 0.27 & 0 & 0 & 0.27 & 1 & 0.14 & 0.25 & 0.27 & 0.14 & 0.25 & 0.27 & 0.24 & 0.31 \\
\hline \begin{tabular}{|l|} 
Russian \\
\end{tabular} & 0.13 & 0.14 & 0.15 & 0.12 & 0.13 & 0.15 & 0 & 0 & 0.15 & 0.14 & 1 & 0.14 & 0.15 & 0.67 & 0.14 & 0.15 & 0.13 & 0.18 \\
\hline Crisis & 0.24 & 0.25 & 0.27 & 0.56 & 0.24 & 0.27 & 0 & 0 & 0.27 & 0.25 & 0.14 & 1 & 0.27 & 0.14 & 0.25 & 0.27 & 0.59 & 0.46 \\
\hline Market & 0.25 & 0.53 & 0.67 & 0.24 & 0.62 & 0.29 & 0 & 0 & 0.29 & 0.27 & 0.15 & 0.27 & 1 & 0.15 & 0.62 & 0.29 & 0.25 & 0.33 \\
\hline Investor & 0.13 & 0.14 & 0.15 & 0.12 & 0.13 & 0.15 & 0 & 0 & 0.15 & 0.14 & 0.67 & 0.14 & 0.15 & 1 & 0.14 & 0.15 & 0.13 & 0.18 \\
\hline Trade & 0.24 & 0.5 & 0.94 & 0.22 & 0.56 & 0.27 & 0 & 0 & 0.27 & 0.25 & 0.14 & 0.25 & 0.62 & 0.14 & 1 & 0.27 & 0.24 & 0.31 \\
\hline Money & 0.25 & 0.27 & 0.29 & 0.24 & 0.25 & 0.43 & 0 & 0 & 0.86 & 0.27 & 0.15 & 0.27 & 0.29 & 0.15 & 0.27 & 1 & 0.25 & 0.33 \\
\hline Hurt & 0.22 & 0.24 & 0.25 & 0.95 & 0.22 & 0.25 & 0 & 0 & 0.25 & 0.24 & 0.13 & 0.59 & 0.25 & 0.13 & 0.24 & 0.25 & 1 & 0.43 \\
\hline Assets & 0.29 & 0.31 & 0.33 & 0.4 & 0.29 & 0.33 & 0 & 0 & 0.33 & 0.31 & 0.18 & 0.46 & 0.33 & 0.18 & 0.31 & 0.33 & $\begin{array}{ll}0.43 \\
\end{array}$ & 1 \\
\hline
\end{tabular}

$(\mathrm{TF}+\mathrm{STF})^{*} \mathrm{IDF}$ for each word contained in document 1 and document 2 respectively. We have also shown in the tables the overall score obtained by each sentence.

In Table 3, STF for the word "trade" is 1, because the word "trade" is highly semantically related to the word "Commerce" as shown in figure 3 and their similarity crosses the similarity threshold value. Since, for our small example, it happens only once, STF for word "trade" is 1 .

\section{Use of Hybrid Similarity in Redundancy Removal Process}

According to sentence scores shown in Table 3 and Table 4, the sentence $S_{1}$ will be selected first because it has the highest overall score. Before selecting the second sentence, we calculate hybrid similarity between sentences $S_{1}$ and $S_{2}$. The similarity calculation is shown in Table 5 .

Table 3. TF(term frequency), STF(Semantic TF), (TF+STF)*IDF and overall score calculation for the sentence $\left(\mathrm{S}_{1}\right)$ in document1

\begin{tabular}{|c|c|c|c|c|c|c|c|c|}
\hline Word & $\operatorname{Tf}(\mathbf{w})$ & $\operatorname{Stf}(w)$ & idf & $\begin{array}{c}\text { Weight }(w)= \\
(\mathrm{TF}(w)+\mathrm{STF}(w)) \\
* \operatorname{IDF}(w)\end{array}$ & $\begin{array}{c}\text { Score }_{t}(S) \\
= \\
\sum_{w \in S} w_{e i g h t}(w)\end{array}$ & $\begin{array}{l}\text { Normalized } \\
(A)\end{array}$ & $\begin{array}{l}\text { Score }_{p}(S) \\
=\frac{1}{\sqrt{i}}\end{array}$ & $\begin{array}{l}\text { Overall } \\
\text { Score = } \\
(\text { A) }+(\text { B })\end{array}$ \\
\hline Russian & 2 & 0 & 3.0 & 6.0 & \multirow{10}{*}{62.22} & \multirow[b]{4}{*}{62.22} & \multirow{10}{*}{1.0} & \multirow{10}{*}{1.99} \\
\hline crisis & 1 & 0 & 4.2 & 4.2 & & & & \\
\hline hurt & 1 & 1 & 6.86 & 13.72 & & & & \\
\hline bilateral & 1 & 0 & 4.76 & 4.76 & & & & \\
\hline trade & 1 & 1 & 2.63 & 5.26 & & \multirow{6}{*}{$\max _{=0.99}\{62.22,62.28\}$} & & \\
\hline caused & 1 & 0 & 3.66 & 3.66 & & & & \\
\hline Ukranian & 1 & 0 & 3.0 & 3.0 & & & & \\
\hline currency & 1 & 1 & 4.13 & 8.26 & & & & \\
\hline fall & 2 & 0 & 4.25 & 8.5 & & & & \\
\hline committee & 1 & 0 & 4.86 & 4.86 & & & & \\
\hline
\end{tabular}


Table 4. TF(term frequency), STF(Semantic TF), (TF+STF)*IDF and overall score calculation for the sentence $\left(\mathrm{S}_{2}\right)$ in document2

\begin{tabular}{|c|c|c|c|c|c|c|c|c|}
\hline Word & $\mathbf{T f}(w)$ & Stf(w) & idf & $\begin{array}{c}\text { Weight }(\mathbf{w})= \\
(\operatorname{TF}(w)+\operatorname{STF}(w)) \\
* \operatorname{IDF}(w)\end{array}$ & $\begin{array}{c}\text { Score }_{t}(S) \\
= \\
\sum_{w \in S} \text { weight }(w)\end{array}$ & $\begin{array}{c}\text { Normalized } \\
(A)\end{array}$ & $\begin{array}{c}\text { Score }_{p}(S) \\
\frac{1}{\sqrt{i}} \\
=\frac{(\mathbf{B})}{}\end{array}$ & $\begin{array}{l}\text { Overall } \\
\text { Score }=\end{array}$ \\
\hline Russian & 2 & 0 & 3.0 & 6.0 & \multirow{10}{*}{62.22} & \multirow{10}{*}{62.28} & \multirow{10}{*}{0.7} & \multirow{10}{*}{1.7} \\
\hline money & 1 & 1 & 3.54 & 7.08 & & & & \\
\hline market & 1 & 0 & 2.41 & 2.41 & & & & \\
\hline investor & 1 & 0 & 4.25 & 4.25 & & & & \\
\hline assets & 1 & 0 & 4.31 & 4.31 & & & & \\
\hline continued & 1 & 0 & 3.1 & 3.1 & & & & \\
\hline fall & 2 & 0 & 4.25 & 8.5 & & & & \\
\hline wound & 1 & 1 & 6.43 & 12.86 & & & & \\
\hline hit & 1 & 0 & 4.73 & 4.73 & & & & \\
\hline commerce & 1 & 1 & 4.52 & 9.04 & & & & \\
\hline
\end{tabular}

Table 5. Hybrid similarity calculation

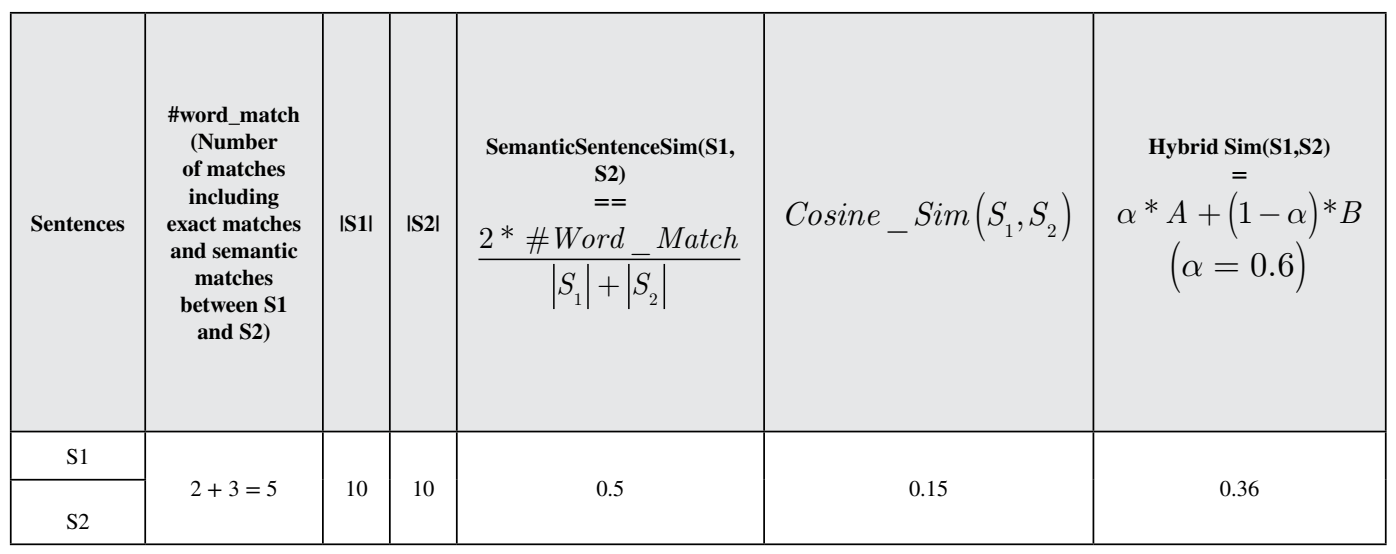

As we can see from Table 5, hybrid similarity between $S_{1}$ and $S_{2}$ is 0.36 which is less than the threshold value. So, $S_{2}$ is considered as sufficiently dissimilar and added to the summary.

\section{Experiments and Results}

We have used DUC 2003 and DUC 2004 benchmark generic multi-document summarization datasets for experimentation and results. The DUC 2003 dataset consists of 30 input folders for multi-document summarization and DUC 2004 dataset consists of 50 input folders. For both the datasets, each input folder consists of approximately 10 news articles. According to DUC specifications, multi-document summarization task (task2) was to generate approximately 100 words ( 665 bytes) summary for each input.

Both the datasets were released by the organizers of DUC (NIST) ${ }^{2}$. For each input folder, four reference summaries created by the four different human summarizers were made available. For evaluation of our system generated summaries, an automatic evaluation tool ROUGE (version 1.5.5) package is used (Lin,2004). 
ROUGE package compares the system generated summary with the set of reference (model) summaries and computes ROUGE-N scores based on how many words N-grams match between the system-generated summaries and the reference summaries. For example, ROUGE-1 score is reported when unigram matches are considered, ROUGE-2 score is reported when bigram overlap is considered and so on. For each such ROUGE score, ROUGE package reports precision, recall and F-score. We have considered ROUGE-1 and ROUGE-2 F-scores for summary evaluation. We have set the option -b 665 (as per specifications of DUC 2004 task2) in the ROUGE package. This option enables the ROUGE package to take the only first 665 bytes of the system summary in the evaluation process.

\section{Results}

To prove effectiveness of the semantic term relations in multi-document text summarization, we have developed two text summarization models - (1) WordNet based summarization model which exploits WordNet based semantic relations in term weighting and sentence similarity measures and (2) Word embedding based Summarization model that exploits word embedding based semantic term relations in term weighting and sentence similarity measures. Both the models have been discussed in the earlier sections.

The basic model parameters are the same for both the models though we have observed that when the datasets and the models are changed, the values of the parameters need to change for the best results. As we mentioned in the earlier sections of this paper, for both the models, the most important model parameters are the three threshold values- (1) threshold on word-to-word similarity value (Say, $\mathrm{T}_{\text {wsim }}$ ). Here word-to-word similarity value is basically Wup value for WordNet based model and cosine similarity value for word embedding based model, (2) threshold on term weight (say, $\mathrm{T}_{\mathrm{tw}}$ ) to exclude low weight terms from the sentence scoring process and (3) threshold on sentence similarity (say, $\mathrm{T}_{\text {ssim }}$ ) used for redundancy removal.

The best configuration for the parameters of the proposed models are shown in Table 6.

We have used DUC 2003 and DUC 2004 task 2 datasets for testing the summarization models. The ROUGE F-scores obtained by our proposed models for DUC 2003 and DUC 2004 datasets respectively are presented in Table 7 and Table 8 .

To prove effectiveness of our proposed word summarization models, we have compared it with two important summarization baseline systems-(1) DUC Coverage baseline which is to generate a summary from the input cluster of documents by taking one by one the first sentence from each document and (2) MEAD baseline (Radev et al., 2004) which is one of hard baselines for generic multi-document summarization. In order to compare MEAD's performance with our proposed summarization system, we have implemented the MEAD system with three important features- centroid, position and length cutoff (Radev et al., 2004).

The ROUGE F-scores obtained by DUC baseline system and MEAD baseline system for DUC 2003 and DUC 2004 datasets are also shown in Table 7 and Table 8 respectively. As can be seen from Table 7 and Table 8, our proposed word embedding based summarization model performs the best among all four models presented in the tables. It is also clear from our obtained results that the WordNet based summarization model does not perform well and this also indicates that WordNet

Table 6. Configuration of model Parameters

\begin{tabular}{|l|l|l|l|}
\hline \multirow{2}{*}{ Methods } & \multicolumn{3}{c|}{ Parameters } \\
\cline { 2 - 4 } & \multicolumn{1}{c|}{$\mathbf{T h}_{\mathrm{wsim}}$} & \multicolumn{1}{c|}{$\mathbf{T}_{\text {tw }}$} & \multicolumn{1}{c|}{$\mathbf{T}_{\text {ssim }}$} \\
\hline WordNet based Summarization model & 0.85 & 5 & 0.4 \\
\hline Word Embedding based Summarization model & 0.7 & 4 & 0.45 \\
\hline
\end{tabular}


Table 7. Comparison Results on DUC 2003 data

\begin{tabular}{|l|l|l|}
\hline \multicolumn{1}{|c|}{ SYSTEMS } & \multicolumn{1}{c|}{ ROUGE-1 F-score } & \multicolumn{1}{c|}{ ROUGE-2 F-score } \\
\hline $\begin{array}{l}\text { Proposed Word Embedding based } \\
\text { Summarization Model }\end{array}$ & 0.3618 & 0.0878 \\
\hline $\begin{array}{l}\text { Proposed WordNet based } \\
\text { Summarization Model }\end{array}$ & 0.3523 & 0.0791 \\
\hline MEAD Baseline & 0.3581 & 0.0873 \\
\hline DUC Coverage baseline & 0.3244 & 0.0717 \\
\hline
\end{tabular}

Table 8. Comparison Results on DUC 2004 data

\begin{tabular}{|l|l|l|}
\hline \multicolumn{1}{|c|}{ SYSTEMS } & \multicolumn{1}{|c|}{\begin{tabular}{c}
\multicolumn{1}{c|}{ ROUGE-1 } \\
F-score
\end{tabular}} & \multicolumn{1}{c|}{ F -score } \\
\hline $\begin{array}{l}\text { Proposed Word Embedding based } \\
\text { Summarization Model }\end{array}$ & 0.3840 & 0.0956 \\
\hline $\begin{array}{l}\text { Proposed WordNet based } \\
\text { Summarization Model }\end{array}$ & 0.3786 & 0.0918 \\
\hline MEAD baseline & 0.3737 & 0.0937 \\
\hline DUC Coverage baseline & 0.3451 & 0.0812 \\
\hline
\end{tabular}

based semantic term relations are less useful than word embedding based term relations in improving multi-document performance.

\section{System Comparison}

To prove whether the proposed approach is effective or not, we conducted statistical significance tests. To do this, we have generated ROUGE scores for each individual system summary using ROUGE package. At first, we did statistical significance test between two groups of ROUGE scores obtained by our two different summarization models- word Embedding based summarization model and WordNet based summarization model. Two tailed paired T-test results shows that the difference of ROUGE-1 F-score obtained by these two models are statistically significant for DUC 2003 dataset $(p<0.05)$, but it is not statistically significant for DUC 2004 dataset $(p=0.22>0.05)$. So, these results indicate that word embedding based semantic term relations are more useful in improving text summarization performance on DUC 2003 dataset.

We have also compared our proposed summarization models with two important summarization baseline systems-(1) DUC Coverage baseline which has been defined earlier in this section and (2) MEAD baseline (Radev et al., 2004) which is one of the hard baselines for generic multi-document summarization. In order to compare MEAD's performance with our proposed summarization systems, we have implemented the MEAD system with three important features- centroid, position and length cutoff (Radev et al., 2004). The results of two tailed paired tests are shown in Table 9. We can conclude from the results of statistical significance test that our proposed system that exploits word embedding based semantic term relations in multi-document text summarization performs significantly better than DUC baseline for both the datasets. For DUC 2004 dataset, the proposed word embedding based approach performs significantly better than the MEAD baseline also. On the other hand, though the WordNet based model performs significantly better than DUC baseline for both the datasets, it cannot perform significantly better than the MEAD baseline. So, the results indicate that leveraging semantic term relations can enhance multi-document text summarization performance. 
Table 9. Results of two tailed paired T-test

\begin{tabular}{|c|c|c|c|}
\hline Datasets & System1 & System 2 & p-value \\
\hline \multirow{4}{*}{ DUC 2003} & $\begin{array}{l}\text { Proposed Word Embedding based Summarization } \\
\text { System }\end{array}$ & MEAD Baseline & $\mathrm{p}>0.05$ \\
\hline & $\begin{array}{l}\text { Proposed Word Embedding based Summarization } \\
\text { System }\end{array}$ & DUC coverage baseline & $\mathrm{p}<0.01$ \\
\hline & $\begin{array}{l}\text { Proposed WordNet based Summarization } \\
\text { System }\end{array}$ & MEAD Baseline & $\mathrm{p}>0.05$ \\
\hline & $\begin{array}{l}\text { Proposed WordNet based Summarization } \\
\text { System }\end{array}$ & DUC coverage baseline & $\mathrm{p}<0.001$ \\
\hline \multirow{4}{*}{ DUC 2004} & $\begin{array}{l}\text { Proposed Word Embedding based Summarization } \\
\text { System }\end{array}$ & MEAD Baseline & $\mathrm{p}<0.002$ \\
\hline & $\begin{array}{l}\text { Proposed Word Embedding based Summarization } \\
\text { System }\end{array}$ & DUC coverage baseline & $\mathrm{p}<0.0001$ \\
\hline & $\begin{array}{l}\text { Proposed WordNet based Summarization } \\
\text { System }\end{array}$ & MEAD Baseline & $\mathrm{p}>0.05$ \\
\hline & $\begin{array}{l}\text { Proposed WordNet based Summarization } \\
\text { system }\end{array}$ & DUC coverage baseline & $\mathrm{p}<0.0001$ \\
\hline
\end{tabular}

\section{Error Analysis}

We have randomly selected some bad summaries and good summaries generated by our proposed word embeddings-based model and inspected the generated summaries. Finally, we have identified that the bad summaries were generated for the cases when the chosen summary sentence are relatively longer and the longer sentences contain many words which do not match with the reference summaries which are basically human created abstracts. Since such sentences cannot be discarded because of its portion containing many important words, it occupies major part of summary space constrained by the predefined summary length restriction and prevents other sentences from being selected in the summary. We also observe that, in spite of incorporating semantic word matching in detecting and removing redundancy, the generated summaries still contain some redundancy.

Our obtained results indicate that word embedding based semantic word relations are more useful in text summarization than WordNet based semantic word relations. The main problem with the WordNet based semantic word relations is that WordNet maintains various types of word relations such as Synonymy, hypernymy, holonomy, antonym etc. If two words are completely opposite in meaning, WordNet based similarity measure such as WUP and Lin shows very high value. But for summarization task, we need to compute how many times a word is semantically similar with other words. Though, to combat this situation, we tried to capture such relations by setting the higher threshold on Wup value, merely setting the higher threshold on Wup value could not give the expected results.

\section{CONCLUSION AND FUTURE WORK}

In this study, we have presented a multi-document summarization technique that exploits semantic term relations in term weighting and redundancy removal. According to the experimental results and system comparison using statistical significance tests, our proposed summarizer performs significantly better than several baselines.

After analyzing the results obtained by our proposed summarizer and comparing it with the baseline summarizers, we can conclude that: (1) leveraging distributional semantics based term relations can improve performance of the summarizer (2) combining semantic similarity measure 
with syntactic sentence similarity measure is effective in reducing redundancy in extractive summary and (3) distributional semantics based term relations are more useful in improving text summarization performance than WordNet based semantic term relations. But the primary disadvantage of distributional semantics-based summarization model is that it is more computationally expensive than the simple term frequency-based baseline summarization systems.

Our future work will include hybridization of multiple semantic models for capturing semantic term relations in more accurate and effective manner in order to improve ranking algorithm and redundancy removal algorithm for producing better quality summaries.

\section{ACKNOWLEDGMENT}

This work has received support from the project titled "Indian Social Media Sensor: an Indian Social Media Text Mining System for Topic Detection, Topic Sentiment Analysis and Opinion Summarization' funded by the Department of Science and Technology (DST)[grant number EEQ/2017/000369], Government of India under the SERB scheme. 


\section{REFERENCES}

Al-Sabahi, K., \& Zuping, Z. (2019). Document summarization using sentence-level semantic based on word embeddings. International Journal of Software Engineering and Knowledge Engineering, 29(02), $177-196$. doi:10.1142/S0218194019500086

Aliguliyev, R. M. (2009). A new sentence similarity measure and sentence based extractive technique for automatic text summarization. Expert Systems with Applications, 36(4), 7764-7772. doi:10.1016/j.eswa.2008.11.022

Belwal, R. C., Rai, S., \& Gupta, A. (2020). A new graph-based extractive text summarization using keywords or topic modeling. Journal of Ambient Intelligence and Humanized Computing, 1-16. doi:10.1007/s12652020-02591-x

Budanitsky, A., \& Hirst, G. (2006). Evaluating WordNet-based measures of lexical semantic relatedness. Computational Linguistics, 32(1), 13-47. doi:10.1162/coli.2006.32.1.13

Carbonell, J. G., \& Goldstein, J. (1998). The use of MMR, diversity-based re-ranking for reordering documents and producing summaries. Proceedings of the 21st Annual International ACM SIGIR Conference on Research and Development in Information Retrieval, 335-336.

Dam, S., \& Sarkar, K. (2019). Using WordNet-based Semantic Relatedness Measure for Reducing Redundancy and Improving Multi-document Text Summarization. International Journal on Computer Science and Engineering, 07(01), 268-273.

Dam, S., Sarkar, K., \& Chowdhury, S. (2018). A Study on Effect of Positional Information in Single Document Text Summarization. International Journal of Computers and Applications, 13(1), 29-37.

Erkan, G., \& Radev, D. R. (2004). LexRank: Graph-based lexical centrality as salience in text summarization. Journal of Artificial Intelligence Research, 22, 457-479. doi:10.1613/jair.1523

Fellbaum, C. (1998). WordNet: An Electronic Lexical Database. The MIT Press.

Goldstein, J., Mittal, V., Carbonell, J., \& Kantrowitz, M. (2000). Multi-document summarization by sentence extraction. Proceedings of Conference of NAACL-ANLP-AutoSum '00: 2000 NAACL-ANLP Workshop on Automatic summarization, 4.

Gupta, V. K., \& Siddiqui, T. J. (2012). Multi-document summarization using sentence clustering. Proceedings of 4th International Conference on Intelligent Human Computer Interaction (IHCI), IHCI 2012, 1-5. . 1-5. doi:10.1109/IHCI.2012.6481826

Hardy, H., Shimizu, N., Strzalkowski, T., Ting, L., Wise, G. B., \& Zhang, X. (2002). Cross-document summarization by concept classification. Proceedings of the 25th Annual International ACM SIGIR Conference on Research and Development in Information Retrieval, 121-128.

Hatzivassiloglou, V., Klavans, J. L., Holcombe, M. L., Barzilay, R., Kan, M.-Y., \& McKeown, K. R. (2001). SimFinder: A Flexible Clustering Tool for Summarization. NAACL, Workshop on Automatic Summarization, Pittsburgh, PA.

Lin, C. Y. (2004). ROUGE: A package for automatic evaluation of summaries. WAS 2004: Proceedings of the Workshop on Text Summarization Branches Out. https://aclanthology.org/W04-1013

Lin, D. (1998, July). An information-theoretic definition of similarity. ICML, 98, 296-304.

Luhn, H. P. (1958). The automatic creation of literature abstracts. IBM Journal of Research and Development, 2(2), 159-165. doi:10.1147/rd.22.0159

Mihalcea, R. \& Tarau, P. (2004). TextRank: Bringing order into text. Proceedings of the 2004 Conference on Empirical Methods in Natural Language Processing, 404-411.

Mihalcea, R., \& Tarau, P. (2005). A language independent algorithm for single and multiple document summarization. Proceedings of IJCNLP 2005, Companion Volume to the Proceedings of Conference including Posters/Demos and tutorial abstracts.

Mikolov, T., Sutskever, I., Chen, K., Corrado, G. S., \& Dean, J. (2013). Distributed representations of words and phrases and their compositionality. Advances in neural information processing systems, 3111-3119. 
Mohd, M., Jan, R., \& Shah, M. (2020). Text document summarization using word embedding. Expert Systems with Applications, 143, 112958. doi:10.1016/j.eswa.2019.112958

Nallapati, R., Zhai, F., \& Zhou, B. (2017). SummaRunner: A recurrent neural network based sequence model for extractive summarization of documents. Proceedings of the Thirty-First AAAI Conference on Artificial Intelligence.

Nallapati, R., Zhou, B., dos Santos, C., Gulcehre, C., \& Xiang, B. (2016). Abstractive text summarization using sequence to-sequence RNNS and beyond. In Proceedings of the 20th SIGNLL Conference on Computational Natural Language Learning. Association for Computational Linguistics. doi:10.18653/v1/K16-1028

Radev, D. R., Allison, T., Blair-Goldensohn, S., Blitzer, J., Celebi, A., Dimitrov, S., Drabek, E., Hakim, A., Lam, W., Liu, D., \& Otterbacher, J. (2004). MEAD-A Platform for Multidocument Multilingual Text Summarization. Proceedings of the Fourth International Conference on Language Resources and Evaluation published in ELRA.

Radev, D. R., Jing, H., Stys, M., \& Tam, D. (2004). Centroid-based summarization of multiple documents. Information Processing \& Management, 40(6), 919-938. doi:10.1016/j.ipm.2003.10.006

Resnik, P. (1995). Using information content to evaluate semantic similarity. Proceedings of the 14th International Joint Conference on Artificial Intelligence, 448-453.

Rush, A., Chopra, S., \& Weston, J. (2015). A Neural Attention Model for Abstractive Sentence Summarization. Proceedings of EMNLP. arXiv:1509.00685.

Sarkar, K. (2009a). Sentence clustering-based summarization of multiple text documents. Int. J. Comput. Sci. and Commun. Tech, 2(1), 225-235.

Sarkar, K. (2009b). Using domain knowledge for text summarization in medical domain. International Journal of Recent Trends in Engineering, 1(1), 200-205.

Sarkar, K. (2014). A Keyphrase-Based Approach to Text Summarization for English and Bengali Documents. International Journal of Technology Diffusion, 5(2), 28-38. doi:10.4018/ijtd.2014040103

Sarkar, K., \& Bandyopadhyay, S. (2005). Generating headline summary from a document set. Proceedings of International Conference on Intelligent Text Processing and Computational Linguistics, 649-652. doi:10.1007/978-3-540-30586-6_72

Sarkar, K., Nasipuri, M., \& Ghosh, S. (2011). Using machine learning for medical document summarization. International Journal of Database Theory and Application., 4, 31-49.

Sarkar, K., Saraf, K., \& Ghosh, A. (2015). Improving graph based multidocument text summarization using an enhanced sentence similarity measure. IEEE 2 nd International Conference on Recent Trends in Information Systems (ReTIS), 359-365. doi:10.1109/ReTIS.2015.7232905

Song, Y. I., Han, K. S., \& Rim, H. C. (2004). A term weighting method based on lexical chain for automatic summarization. Proceedings of International Conference on Intelligent Text Processing and Computational Linguistics, 636-639. doi:10.1007/978-3-540-24630-5_78

Uçkan, T., \& Karcı, A. (2020). Extractive multi-document text summarization based on graph independent sets. Egyptian Informatics Journal., 21(3), 145-157. doi:10.1016/j.eij.2019.12.002

Wu, Z., \& Palmer, M. (1994). Verb semantics and lexical selection. Proceedings of the 32nd Annual meeting of the Associations for Computational Linguistics, 133-138.

Zajic, D., Dorr, B., \& Schwartz, B. (2002). Automatic Headline Generation for Newspaper Stories. Proceedings of the ACL Workshop on Automatic Summarization (DUC), 78-85.

\section{ENDNOTES}

http://mattmahoney.net/dc/text8.zip retrieved in 2019

http://duc.nist.gov 
Kamal Sarkar received his B.E degree in Computer Science and Engineering from the Faculty of Engineering, Jadavpur University in 1996. He received the M.E degree and Ph.D. (Engg) in Computer Science and Engineering from the same University in 1999 and 2011 respectively. In 2001, he joined as a lecturer in the Department of Computer Science \& Engineering, Jadavpur University, Kolkata, where he is currently an associate professor. His research interest includes text summarization, natural language processing, machine learning, web mining, knowledge discovery from text data.

Santanu Dam Completed M. Tech from Jadavpur University, India in 2010. He is currently pursuing Ph.D. and working as Deputy Director at Netaji Subhas Open University. His main research work focuses on Natural Language Processing, Machine Learning, Text Summarization, Text Mining, Cloud Computing. He has 14 years of teaching experience. 